\title{
Photoelectrochemistry: From Nanomaterials to Devices
}

\section{Laurie Peter}

Following its development as a part of semiconductor electrochemistry, the science of Photoelectrochemistry has expanded steadily to cover a wide range of materials and processes, ranging from photocatalysis in dispersions of colloidal metal oxides, through studies of well-defined surfaces of semiconductor single crystals to nanostructured semiconductors used in dye-sensitized solar cells and water splitting systems. It would be impossible to introduce the topic of Photoelectrochemistry without mentioning the pivotal role of the (by now classical) Nature paper by Fujishima and Honda on the splitting of water by sunlight at single crystal titania, which appeared 35 years ago. This was a year before the 1973 oil crisis, which saw the price of oil increase from $3 \$$ /barrel to more than $\$ 12 /$ barrel. With oil currently topping $100 \$$ /barrel, these changes may seem small in the new millennium, but the shock waves that spread through the world economy in the 1970's stimulated an upsurge of interest in solar energy conversion that led to remarkably rapid advances in photoelectrochemistry, producing a range of high efficiency photoelectrochemical devices for solar energy conversion and also laying the theoretical and experimental foundations of the subject. This exciting period marks the birth of modern Photoelectrochemistry.

Ultimately, exploitation of these new photoelectrochemical concepts for solar energy conversion was prevented by concerns about the long term stability of the semiconductor materials under operating conditions. As a consequence, interest in photoelectrochemistry declined slowly during the 1980s before being stimulated again by a second Nature paper, this time by O'Regan and Grätzel, who described a new type of photoelectrochemical solar cell based on the sensitization of nanocrystalline titania layers by a ruthenium-based dye. The dye-sensitized solar cell avoids electrode stability problems of the type encountered in the previous generation of regenerative photoelectrochemical solar cells, although we are still waiting for the cells to leave the laboratory and enter the marketplace.

Japanese research groups have been at the forefront of many of these developments in Photoelectrochemistry. Research in Japan on dye-sensitized solar cells has been particularly intense over the last decade, and world-record results have been achieved along with many valuable innovations and insights. So it is clear that Photoelectrochemistry is still alive and well 30 years after Fujishima and Honda's key paper, and this Special Issue of Electrochemistry provides a timely illustration of the broad range and high quality of work being carried out in Japan.

Interestingly, the balance of papers in this issue shows that Photoelectrochemistry is no longer focussed almost entirely on solar energy conversion. Whilst there are several papers that deal with dye-sensitized solar cells, single crystal silicon is well represented with papers returning to the still relevant problems of metal electrodeposition and etching. Environmental applications are also covered, with several papers on different aspects of photocatalysis at semiconductor nanoparticles. Photoelectrochemistry has clearly recovered its original wider appeal, and the next decade is likely to see emphasis on new applications in addition to the continuing focus on photoelectrochemical approaches to utilization of the power of sunlight. The future for Photoelectrochemistry remains bright.

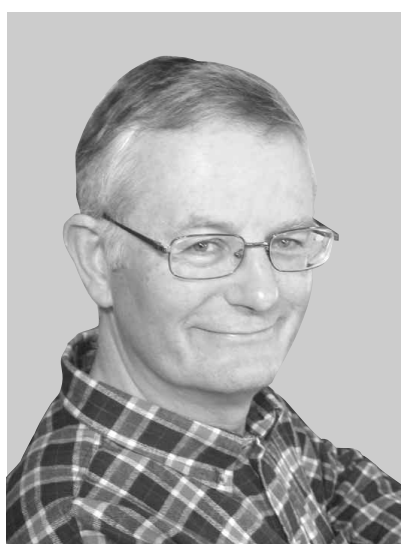

Laurie PETER Professor, University of Bath 